\title{
EXCEPTIONAL SETS FOR LINEAR DIFFERENTIAL POLYNOMIALS
}

\author{
J. K. LANGLEY
}

\section{Introduction}

Let $f(z)$ be a non-constant entire function with Nevanlinna characteristic $T(r, f)$ (see e.g. [7]). Suppose that

$$
\psi(z)=\sum_{i=0}^{k} \alpha_{i}(z) f^{(i)}(z)
$$

is non-constant, where $\alpha_{0}(z), \ldots, \alpha_{k}(z)$ are entire functions each satisfying

$$
T\left(r, \alpha_{i}\right)=S(r, f)
$$

and, using standard notation from [7], $S(r, f)$ denotes any quantity such that

$$
S(r, f)=o(T(r, f)) \text {, }
$$

possibly outside a set of finite linear measure. Then we have the following bound on the growth of $T(r, f)$ ([7], p. 57):

Theorem A. If $f(z)$ is a non-constant entire function, and if $\psi(z)$ is given by (1.1) and (1.2) and is non-constant, then

$$
T(r, f)<N\left(r, \frac{1}{f}\right)+N\left(r, \frac{1}{\psi-1}\right)-N\left(r, \frac{1}{\psi^{\prime}}\right)+S(r, f) .
$$

We observe that Theorem $\mathbf{A}$ is usually stated in a slightly different form, with common zeros of $\psi(z)-1$ and $\psi^{\prime}(z)$ cancelled out, but the statement (1.3) is more convenient for our purposes here.

It follows from Theorem A (Hayman [5]) that if $g(z)$ is a transcendental entire function, and $N \geqq 2$, then $g^{N}(z) g^{\prime}(z)$ has infinitely many 1-points. It was shown in [2] by Anderson, Baker and Clunie that infinitely many of these 1-points must lie outside certain exceptional sets. They proved:

Theorem B. If $\left(a_{n}\right)$ is a sequence converging to infinity such that, for all $n$,

$$
\left|\frac{a_{n+1}}{a_{n}}\right|>q>1
$$

Supported in part by NSF grant MCS82-00497. 
then for any $N \geqq 2$ every transcendental entire function $g(z)$ must have infinitely many solutions of

outside $E=\left\{a_{n}\right\}$.

$$
g^{N}(z) g^{\prime}(z)=1
$$

This result was improved in the author's Ph. D. thesis [8], written under the supervision of I. N. Baker, in which it is shown that the exceptional set $E$ may consist of a countable union of small discs whose centres $a_{n}$ satisfy (1.4). These exceptional sets are comparable to certain Picard sets for entire functions (see [3], [4], [10]) - that is, subsets of the plane outside which every transcendental entire function takes every finite value, with at most one exception, infinitely often.

In the present paper we return to the initial problem of Theorem $A$ in the case where $\alpha_{0}(z), \ldots, \alpha_{k}(z)$ are polynomials and $\alpha_{k}(z) \not \equiv 0$. We prove:

Theorem 1. Given $\varepsilon>0$, there exists $K(\varepsilon)>0$, depending only on $\varepsilon$, such that if $\left(a_{n}\right)$ converges to infinity with

$$
\left|a_{n}-a_{m}\right|>\varepsilon\left|a_{n}\right|
$$

for all $n \neq m$, while $\left(\varrho_{n}\right)$ satisfies

$$
\log \frac{1}{\varrho_{n}}>K(\varepsilon)\left(\log \left|a_{n}\right|\right)^{2}
$$

then for any polynomials $\alpha_{0}(z), \ldots, \alpha_{k}(z)$ (with $k \geqq 1$ and $\alpha_{k}(z) \not \equiv 0$ ) and any transcendental entire function $f(z)$ such that

$$
\psi(z)=\sum_{i=0}^{k} \alpha_{i}(z) f^{(i)}(z)
$$

is non-constant, either $f(z)$ has infinitely many zeros outside the union $E$ ot the discs

$$
B\left(a_{n}, \varrho_{n}\right)=\left\{z:\left|z-a_{n}\right|<\varrho_{n}\right\}
$$

or $\psi(z)$ has infinitely many 1-points outside $E$.

The condition (1.5) is best-possible. We have, in fact:

Theorem 2. Given any increasing, positive function $h(r)$ on $0<r<\infty$, such that $h(r) \rightarrow \infty$ as $r \rightarrow \infty$, there exists a transcendental entire function $f(z)$ such that all large zeros of $f(z)$ and 1-points of $f^{\prime}(z)$ lie in a set $\left\{b_{m}\right\}$, where, for all $m, b_{m}$ is positive and

$$
b_{m+1} \geqq\left(1+\frac{1}{h\left(b_{m}\right)}\right) b_{m} .
$$

While the exceptional sets of Theorem 1 are comparable to certain Picard sets for entire functions (see [3]), Theorem 2 marks a departure from the situation there, insofar as the best-possible condition on the centres $a_{m}$ of the exceptional discs of 
a Picard set for entire functions is

for $n \neq m$ (Toppila [10]).

$$
\left|a_{n}-a_{m}\right|>\varepsilon\left|a_{n}\right|\left(\log \left|a_{n}\right|\right)^{-1}
$$

Theorem 2 was proved in the author's Ph. D. thesis [8], while Theorem 1 is a substantial improvement of a result in [8] but makes considerable use of ideas communicated to the author by I. N. Baker.

Throughout the paper we use standard notation from [7], including

$$
M(r, f)=\max \{|f(z)|:|z|=r\}
$$

for an entire function $f(z)$.

\section{Preliminary lemmas}

Lemma 1. Suppose that $P(z)$ is a polynomial of degree $k$ whose zeros $\alpha_{1}, \ldots, \alpha_{k}$ lie in $|z|<R_{0}$. Then for $|z|=R \geqq 3 R_{0}$,

for $i=1, \ldots, k$.

$$
\frac{k !}{(k-i) ! 2^{i} R^{i}} \leqq\left|\frac{P^{(i)}(z)}{P(z)}\right| \leqq \frac{k ! 2^{i}}{(k-i) ! R^{i}}
$$

Proof. We proceed by induction on $i$. We have, for $|z|=R \geqq 3 R_{0}$,

But, for $|t|<1$,

$$
\frac{P^{\prime}(z)}{P(z)}=\frac{1}{z} \sum_{j=1}^{k}\left(1-\frac{\alpha_{j}}{z}\right)^{-1} \text {. }
$$

and so

$$
\operatorname{Re}\left((1-t)^{-1}\right) \geqq 1-\frac{|t|}{1-|t|}
$$

and

$$
\operatorname{Re}\left(\sum_{j=1}^{k}\left(1-\frac{\alpha_{j}}{z}\right)^{-1}\right) \geqq k / 2
$$

$$
\left|\frac{P^{\prime}(z)}{P(z)}\right| \geqq \frac{k}{2 R} \text {. }
$$

For an inequality in the other direction, we just note that if $R=|z| \geqq 3 R_{0}$, then $\left|z-\alpha_{j}\right| \geqq 2|z| / 3$ and so

$$
\left|\frac{P^{\prime}(z)}{P(z)}\right| \leqq \frac{2 k}{R}
$$

To prove the general case, we note that if $1 \leqq i \leqq k-1$,

$$
\frac{P^{(i+1)}(z)}{P(z)}=\frac{P^{(i)}(z)}{P(z)} \frac{P^{(i+1)}(z)}{P^{(i)}(z)}
$$


and since $P^{(i)}(z)$ has $k-i$ zeros, all lying in the convex hull of the set of zeros of $P(z)$ ([1], p. 29), and hence in $|z|<R_{0}$, we have, for $z, R$ as above,

$$
\frac{(k-i)}{2 R} \leqq\left|\frac{P^{(i+1)}(z)}{P^{(i)}(z)}\right| \leqq \frac{2(k-i)}{R}
$$

and Lemma 1 follows by induction.

Lemma 2. Suppose that $R \geqq 1$ and that $h(z)$ is regular and non-zero in $|z| \leqq R$, with $|\log | h(z) \| \leqq M$ on $|z|=R$. Then in $|z| \leqq R / 4$ we have for $i=1, \ldots, k$,

$$
\left|\frac{h^{(i)}(z)}{h(z)}\right| \leqq \beta_{k}(1+M)^{i}
$$

where $\beta_{k}$ depends only on $k$.

Proof. We have (see [7], p. 22)

$$
\frac{h^{\prime}(z)}{h(z)}=\frac{1}{\pi} \int_{0}^{2 \pi} \log \left|h\left(R e^{i \varphi}\right)\right| \frac{\operatorname{Re}^{i \varphi}}{\left(\operatorname{Re}^{i \varphi}-z\right)^{2}} d \varphi
$$

in $|z|<R$, and so

$$
\left|\frac{h^{\prime}(z)}{h(z)}\right| \leqq \beta_{1} M
$$

in $|z| \leqq R / 2$. But (see [7], p. 73), $h^{(i)}(z) / h(z)$ is a differential polynomial in $h^{\prime}(z) / h(z)$ with constant coefficients and total degree at most $i$, and so Lemma 2 follows from (2.1) and Cauchy's estimate.

Lemma 3. Let $N$ and $K$ be positive integers, and suppose that $\left(a_{n}\right)$ is a complex sequence converging to infinity such that, for some $\varepsilon$ with $0<\varepsilon<1$, we have

$$
\left|a_{n}-a_{m}\right|>\varepsilon\left|a_{n}\right|
$$

for all large $n \neq m$. Suppose that the transcendental entire function $h(z)$ is given by

$$
h(z)=\beta z^{Q} \prod_{j=1}^{\infty}\left(1-\frac{z}{\gamma_{j}}\right)
$$

where $\beta \neq 0, Q \geqq 0$ and each $\gamma_{j}$ lies in some disc $B\left(a_{n}, 1\right)$, while, for large $n, B\left(a_{n}, 1\right)$ contains at most $K$ zeros of $h(z)$, counting multiplicities. Then there exist $M_{1}, M_{2}$ depending on $N, K$ and the sequence $\left(a_{n}\right)$, but not on $h(z)$, such that if $|z|=r>M_{1}$ and $n(r / 6,1 / h)>M_{2}$ and $z$ lies outside the union of the discs $B\left(a_{n}, \varepsilon\left|a_{n}\right| / 4\right)$ we have, for $k=1, \ldots, N$,

where

$$
\left(\frac{n(r)}{3 r}\right)^{k} \leqq\left|\frac{h^{(k)}(z)}{h(z)}\right| \leqq\left(\frac{3 n(r)}{r}\right)^{k}
$$

$$
n(r)=n(r, 1 / h) \text {. }
$$


In addition, if $\alpha_{0}(z), \ldots, a_{N}(z)$ are polynomials, not all identically zero, then

satisfies

$$
L(z)=\sum_{k=0}^{N} \alpha_{k}(z) h^{(k)}(z) / h(z)
$$

$$
|L(z)|>\frac{1}{r^{N}}
$$

for $z$ and $r$ as above, provided that $r>M_{3}$, where $M_{3}$ depends only on $\alpha_{0}(z), \ldots, \alpha_{N}(z)$.

Proof. For large $w$ lying outside the union of the discs $B\left(a_{n}, \varepsilon\left|a_{n}\right| / 4\right)$ we first prove by induction on $k$ that

$$
\left(\frac{n(r)}{3 r}\right)^{k} \leqq\left|\frac{h^{(k)}(z)}{h(z)}\right| \leqq\left(\frac{3 n(r)}{r}\right)^{k}
$$

for $|z|=r$ and $|z-w| \leqq \varepsilon|w| 2^{-k-4}$. Now the disc $B(w, \varepsilon|w| / 16)$ does not meet any of the discs $B\left(a_{n}, 1\right)$. Moreover, since the discs $B\left(a_{n}, \varepsilon\left|a_{n}\right| / 2\right)$ are disjoint for large $n$, we see that for any large $R$ and any points $a_{n}$ lying in the annulus

$$
A_{R}=\{\zeta: R \leqq|\zeta| \leqq 2 R\}
$$

there exist corresponding disjoint discs of radius $\varepsilon R / 4$ contained entirely in $A_{R}$, with $a_{n}$ on their respective boundaries, and hence, since $A_{R}$ has area $3 \pi R^{2}$, the number of points $a_{n}$ in $A_{R}$ is at most $48 \varepsilon^{-2}$. Thus, in $B(w, \varepsilon|w| / 32)$, we have, with $|z|=r$,

$$
\frac{h^{\prime}(z)}{h(z)}=\Sigma_{1} \frac{1}{z-\gamma_{j}}+\Sigma_{2} \frac{1}{z-\gamma_{j}}+O\left(\frac{1}{r}\right)
$$

where $\Sigma_{1}$ denotes the sum over all $j$ with $\left|\gamma_{j}\right| \leqq|w| / 6$ and $\Sigma_{2}$ denotes the sum over all $j$ with $\left|\gamma_{j}\right| \geqq 3|w|$. But

$$
\left|\Sigma_{2}\left(z-\gamma_{j}\right)^{-1}\right| \leqq \frac{1}{r} \Sigma_{2}\left|\left(1-\frac{\gamma_{j}}{z}\right)^{-1}\right|<\frac{49 K}{\varepsilon^{2} r} \sum_{i=1}^{\infty}\left(2^{i}-1\right)^{-1}=O\left(\frac{1}{r}\right) .
$$

Also, from Lemma 1,

$$
\left|\Sigma_{1}\left(z-\gamma_{j}\right)^{-1}\right| \geqq \frac{1}{2 r} n(|w| / 6)
$$

and combining (2.3), (2.4) and (2.5), and noting that $n(|w| / 6)$ and $n(r)$ differ by a bounded quantity, we obtain the left-hand inequality of (2.2) in the case $k=1$. Indeed, for $k=1,(2.2)$ holds with 3 replaced by $5 / 2$, the second inequality following from (2.3), (2.4) and the fact that

by Lemma 1.

$$
\left|\Sigma_{1}\left(z-\gamma_{j}\right)^{-1}\right| \leqq \frac{2}{r} n(|w| / 6),
$$


Now, for $k \geqq 1$,

$$
\frac{h^{(k+1)}(z)}{h(z)}=\frac{h^{(k)}(z)}{h(z)} \frac{h^{\prime}(z)}{h(z)}+\frac{d}{d z}\left(\frac{h^{(k)}(z)}{h(z)}\right)
$$

and since (2.2) implies that

$$
\frac{d}{d z}\left(\frac{h^{(k)}(z)}{h(z)}\right)=O\left(\frac{(n(r))^{k}}{r^{k+1}}\right)
$$

in $|z-w| \leqq \varepsilon|w| 2^{-k-5}$, using Cauchy's estimate, we obtain the first part of the lemma by induction.

To prove the second part, suppose that the polynomials $\alpha_{0}(z), \ldots, \alpha_{N}(z)$ have degrees $D_{0}, \ldots, D_{N}$, respectively, and suppose that $z=r e^{i \theta}$ and $n(r / 6,1 / h)$ are such that (2.2) holds for $k=1, \ldots, N$. Set

and

$$
d_{i}=D_{i}-i
$$

and define $s, t$ by

$$
d=\max \left\{d_{i}: \alpha_{i}(z) \not \equiv 0\right\}
$$

and

$$
s=\max \left\{i: d_{i}=d \text { and } \alpha_{i}(z) \not \equiv 0\right\}
$$

$$
t=\max \{s-1,0\} \text {. }
$$

Then, assuming that $r$ is so large that

for each $i$ we write

$$
\log \left|\alpha_{i}(z)\right|=(1+o(1)) D_{i} \log r
$$

$$
L(z)=\Sigma_{3} \alpha_{j}(z) \frac{h^{(j)}(z)}{h(z)}+\Sigma_{4} \alpha_{j}(z) \frac{h^{(j)}(z)}{h(z)}
$$

where $\Sigma_{3}$ denotes the sum over all $j$ with $d_{j}=d$ and $\Sigma_{4}$ denotes the sum over the remaining $j$. But then, using (2.2) we have

$$
\Sigma_{4} \alpha_{j}(z) \frac{h^{(j)}(z)}{h(z)}=O\left((n(r))^{N} r^{d-1}\right)
$$

while, for some $c>0$,

$$
\left|\Sigma_{3} \alpha_{j}(z) \frac{h^{(j)}(z)}{h(z)}\right|>c r^{d}(n(r))^{s}-t O\left(r^{d}(n(r))^{t}\right) .
$$

Since $n(r) \rightarrow \infty$ but satisfies

$$
n(r)=O(\log r)
$$

we obtain the second part of Lemma 3 , noting that $d \geqq-N$.

Lemma 4. Suppose that $q>81$ and that

$$
f(z)=\prod_{n=1}^{\infty}\left(1-\frac{z}{a_{n}}\right)
$$


where $a_{n+1}>a_{n}$ and each $a_{n}$ lies in the set $\left\{q, q^{2}, q^{3}, \ldots\right\}$. Then, for large $n$,

$$
\min \left\{|f(z)|:|z|=q^{1 / 2} a_{n}\right\}>M\left(q^{1 / 4} a_{n}, f\right) .
$$

Proof. For $|z|=q^{1 / 2}\left|a_{N}\right|$ and $n \leqq N$,

while, if $|w|=q^{1 / 4} a_{N}$,

$$
\left|1-\frac{z}{a_{n}}\right| \geqq q^{1 / 2} \frac{a_{N}}{a_{n}}-1
$$

Now,

$$
\left|1-\frac{w}{a_{n}}\right| \leqq q^{1 / 4} \frac{a_{N}}{a_{n}}+1
$$

$$
\frac{q^{1 / 2} \frac{a_{N}}{a_{n}}-1}{q^{1 / 4} \frac{a_{N}}{a_{n}}+1}=\frac{q^{1 / 2} a_{N}-a_{n}}{q^{1 / 4} a_{N}+a_{n}} \geqq\left(q^{1 / 2}-1\right)\left(q^{1 / 4}+1\right)^{-1}>2 .
$$

Also, if $|z|=q^{1 / 2} a_{N}$,

$$
\begin{aligned}
\left|\prod_{n>N}\left(1-\frac{z}{a_{n}}\right)\right| & \geqq \prod_{n>N}\left(1-q^{1 / 2} \frac{a_{N}}{a_{n}}\right) \\
& \geqq \prod_{n=1}\left(1-q^{1 / 2-n}\right)=C_{1},
\end{aligned}
$$

say. On the other hand, for $|w|=q^{1 / 4} a_{N}$,

say. Thus

$$
\begin{aligned}
\sum_{n>N} \log \left|1-\frac{w}{a_{n}}\right| & \leqq \sum_{n>N} \log \left(1+q^{1 / 4} \frac{a_{N}}{a_{n}}\right) \\
& \leqq q^{1 / 4} \sum_{n>N} \frac{a_{N}}{a_{n}} \\
& \leqq q^{1 / 4}\left(\frac{1}{q}+q^{-2}+q^{-3}+\ldots\right) \\
& =C_{2},
\end{aligned}
$$

$$
\left(\min \left\{|f(z)|:|z|=q^{1 / 2} a_{N}\right\}\right)\left(M\left(q^{1 / 4} a_{N}, f\right)\right)^{-1} \geqq 2^{N} C_{1} e^{-C_{2}}>1
$$

for sufficiently large $N$.

We need also a result of Hayman [6]:

Theorem C. Suppose that $f(z)$ is a non-constant entire function satisfying

Then

$$
T(r, f)=O(\log r)^{2} .
$$

$$
\log \left|f\left(r e^{i \theta}\right)\right| \sim \log M(r, f)
$$

as $z=r e^{i \theta}$ tends to infinity outside an $\varepsilon$-set surrounding the large zeros of $f(z)$.

An $\varepsilon$-set is defined (following Hayman [6]) to be a countable set of discs not meeting the origin which subtend angles at the origin whose sum is finite. 


\section{Proof of Theorem 1}

Suppose that $f(z)$ is a transcendental entire function, and that

$$
\psi(z)=\sum_{i=0}^{k} \alpha_{i}(z) f^{(i)}(z)
$$

is non-constant, where $\alpha_{0}, \ldots, \alpha_{k}$ are polynomials, with $\alpha_{k}(z) \not \equiv 0$, and $k \geqq 1$. Suppose that all but finitely many zeros of $f(z)$ and 1-points of $\psi(z)$ lie in the union of the discs $D_{n}=B\left(a_{n}, \varrho_{n}\right)$ where $a_{n} \rightarrow \infty$ such that $\left|a_{n}-a_{m}\right|>\varepsilon\left|a_{n}\right|$ for some $\varepsilon>0$ and for all $n \neq m$, while $\varrho_{n}$ satisfies

$$
\log \frac{1}{\varrho_{n}}>K(\varepsilon)\left(\log \left|a_{n}\right|\right)^{2}
$$

for some $K(\varepsilon)$ which we assume to be large and positive.

We use $c_{1}, c_{2}, \ldots$ to denote positive constants depending only on $\varepsilon$. We first note that (see [7], p. 56)

$$
T(r, \psi) \leqq m(r, \psi / f)+m(r, f) \leqq m(r, f)+S(r, f) .
$$

Also, applying Theorem A,

$$
T(r, f)<N\left(r, \frac{1}{f}\right)+N\left(r, \frac{1}{\psi-1}\right)-N_{1}\left(r, \frac{1}{\psi^{\prime}}\right)+S(r, f),
$$

where $N_{1}\left(r, 1 / \psi^{\prime}\right)$ counts only zeros of $\psi^{\prime}$ which lie in the $\operatorname{discs} B\left(a_{n}, 3 \varrho_{n}\right)$.

We assume, without loss of generality, that $1 / \varepsilon$ and $a_{1}$ are large and that $\left|a_{n}\right| \leqq$ $\left|a_{n+1}\right|$ for all $n$, and observe, as we saw in the proof of Lemma 3 , that the annulus $\left\{z:\left|a_{n}\right| \leqq|z| \leqq 2\left|a_{n}\right|\right\}$ contains less than $49 \varepsilon^{-2}$ of the points $a_{m}$. Thus there exists, for large $n, s_{n}$ satisfying $\left|a_{n}\right| \leqq s_{n} \leqq 2\left|a_{n}\right|$ such that the annulus $\left\{z: s_{n} \leqq|z| \leqq s_{n}+\varepsilon^{2}\left|a_{n}\right| / 50\right\}$ meets none of the discs $B\left(a_{m}, 3 \varrho_{n}\right)$, since $\varrho_{n} \rightarrow 0$. We define $t_{n}, r_{n}$ and the annulus $A_{n}$ by

and

and

$$
t_{n}=s_{n}\left(1+\varepsilon^{2} / 100\right)
$$

$$
r_{n}=s_{n}\left(1+\varepsilon^{2} / 200\right)
$$

Of course, for large $n, A_{n}$ contains no zeros of $f(z)$ or $\psi(z)-1$, nor any zeros of $\psi^{\prime}(z)$ which contribute to $N_{1}\left(r, 1 / \psi^{\prime}\right)$.

We now estimate $T(r, f)$ for large $r$. Let $\mu_{n}$ denote the number of zeros of $f(z)$ in $D_{n}, \sigma_{n}$ the number of 1-points of $\psi(z)$ in $D_{n}$, and $\tau_{n}$ the number of zeros of $\psi^{\prime}$ in $B\left(a_{n}, 3 \varrho_{n}\right)$, in each case counting points according to multiplicity. Suppose that $\hat{r}$ is large, and that the annulus $\left\{z: \beta_{1} \hat{r} \leqq|z| \leqq \beta_{2} \hat{r}\right\}$ meets none of the discs $B\left(a_{n}, 3 \varrho_{n}\right)$, where

and

$$
\beta_{1}=\left(1+\varepsilon^{2} / 200\right)^{-1}
$$

$$
\beta_{2}=\beta_{1}\left(1+\varepsilon^{2} / 100\right) \text {. }
$$


Then, using (3.4), if $\hat{r} \leqq r \leqq \beta_{2} \hat{r}$, we have

$$
\begin{gathered}
T(r, f)<\Sigma^{\prime} \mu_{m}\left(\log \frac{r}{\left|a_{m}\right|}+o(1)\right)+\Sigma^{\prime} \sigma_{m}\left(\log \frac{r}{\left|a_{m}\right|}+o(1)\right) \\
-\Sigma^{\prime} \tau_{m}\left(\log \frac{r}{\left|a_{m}\right|}-o(1)\right)+O(\log r)+S(r, f)
\end{gathered}
$$

where $\Sigma^{\prime}$ denotes the sum over all $m$ with $\left|a_{m}\right|<\beta_{1} \hat{r}$. But

$$
\begin{gathered}
\Sigma^{\prime}\left(\mu_{m}+\sigma_{m}+\tau_{m}\right) \leqq n\left(\beta_{1} \hat{r}, \frac{1}{f}\right)+n\left(\beta_{1} \hat{r}, \frac{1}{\psi-1}\right)+n\left(\beta_{1} \hat{r}, \frac{1}{\psi^{\prime}}\right) \\
\leqq c_{1}\left(N\left(r, \frac{1}{f}\right)+N\left(r, \frac{1}{\psi-1}\right)+N\left(r, \frac{1}{\psi^{\prime}}\right)\right) .
\end{gathered}
$$

Combining (3.5) and (3.7), and using (3.3) and the fact that $f(z)$ is assumed to be transcendental, we obtain, with $r$ as above, and in particular for $r=r_{n}$,

where

$$
T(r, f)<\Sigma^{\prime} y_{m}\left(\log \frac{r}{\left|a_{m}\right|}\right)+S(r, f)
$$

$$
y_{m}=\mu_{m}+\sigma_{m}-\tau_{m} .
$$

Now, whether or not the sequence $\left(y_{m}\right)$ is bounded above, we can find $m_{0}$ and an infinite set $\mathscr{H}$ of $m$ such that

$$
y_{m}=\max \left\{y_{j}: m \geqq j \geqq m_{0}\right\} \geqq 1 .
$$

For $m \in \mathscr{H}$ we choose $M(m)$ satisfying $M(m) \geqq m$ and $\left|a_{M(m)}\right| \leqq s_{m}$ and

$$
y_{M(m)}=\max \left\{y_{j}: j \geqq m \text { and }\left|a_{j}\right| \leqq s_{m}\right\}
$$

and for convenience we set

$$
m^{\prime}=M(m) \text {. }
$$

We note that any absolute bound which holds on $y_{m^{\prime}}$ for $m \in \mathscr{H}$ holds on $y_{m}$ for all large $m$.

For $m \in \mathscr{H}$, we have, from (3.8), (3.9), (3.10) and (3.11),

$$
T(r, f)<M^{\prime} y_{m^{\prime}}\left(\log \frac{r}{\left|a_{1}\right|}\right)+O(\log r)+S(r, f),
$$

for $r_{m} \leqq r \leqq t_{m}$, where $M^{\prime}$ is the number of points $a_{j}$ in $|z|<s_{m}$. Since for large $m, m=O\left(\log \left|a_{m}\right|\right)$ by virtue of the absolute bound on the number of points $a_{n}$ in any annulus $r^{*} \leqq|z| \leqq 2 r^{*}$, we obtain

$$
T\left(r_{m}, f\right)+T\left(r_{m}, \psi\right)<c_{2} y_{m^{\prime}}\left(\log r_{m}\right)^{2} .
$$

We now go on to establish a series of claims and finally to obtain a contradiction if $K(\varepsilon)$ is large enough in (3.2). Suppose that $m$ is large, not necessarily in $\mathscr{H}$, 
and that $z_{1}, \ldots, z_{\mu_{m}}$ are the zeros of $f(z)$ in $D_{m}$, while $w_{1}, \ldots, w_{\sigma_{m}}$ are the 1-points of $\psi(z)$ there. Set

$$
P(z)=\prod_{i=1}^{\mu_{m}}\left(z-z_{i}\right)
$$

and

$$
Q(z)=\prod_{j=1}^{\sigma_{m}}\left(z-w_{j}\right)
$$

and define $h(z), H(z)$ by

$$
f(z)=P(z) h(z)
$$

and

$$
\psi(z)-1=Q(z) H(z)
$$

Claim 1. For any $n, m$, if $\left|a_{m}\right|<r_{n}$, with $r_{n}$ as in (3.5), then

$$
|\log | h(z)|| \leqq c_{3} T\left(r_{n}, f\right)+c_{4} \mu_{m} \log r_{n}
$$

and

$$
|\log | H(z)|| \leqq c_{5} T\left(r_{n}, \psi-1\right)+c_{6} \sigma_{m} \log r_{n}
$$

in $\left|z-a_{m}\right| \leqq 16$.

Applying the Poisson-Jensen formula to $h(z)$ in $|z|<r_{n}$, we obtain (since $\left.|\log x|=\log ^{+} x+\log ^{+}(1 / x)\right)$

$$
|\log | h(z)|| \leqq\left(\frac{r_{n}+|z|}{r_{n}-|z|}\right)\left(m\left(r_{n}, h\right)+m\left(r_{n}, \frac{1}{h}\right)\right)+\sum_{\zeta}^{\prime \prime} \log \left|\frac{r_{n}^{2}-\bar{\zeta}_{z}}{r_{n}(\zeta-z)}\right|
$$

where the sum $\Sigma^{\prime \prime}$ is taken over all zeros of $h$ in $|z|<r_{n}$. But $h(z)$ is non-zero in the disc $B\left(a_{m}, \varepsilon\left|a_{m}\right| / 2\right)$ and so, for $\left|z-a_{m}\right| \leqq 16,(3.20)$ yields

$$
|\log | h(z)|| \leqq c_{7}\left(m\left(r_{n}, h\right)+m\left(r_{n}, \frac{1}{h}\right)\right)+c_{8} n\left(s_{n}, \frac{1}{f}\right) .
$$

Also,

$$
m\left(r_{n}, h\right)+m\left(r_{n}, \frac{1}{h}\right) \leqq m\left(r_{n}, f\right)+m\left(r_{n}, \frac{1}{P}\right)+m\left(r_{n}, \frac{1}{f}\right)+m\left(r_{n}, P\right)
$$

and, noting that $|P(u)| \geqq 1$ on $|u|=r_{n}$, and that

$$
n\left(s_{n}, \frac{1}{f}\right)<c_{9} N\left(r_{n}, \frac{1}{f}\right)
$$

we obtain (3.18) from (3.21). The estimate (3.19) is proved identically.

Claim 2. For all large $m$, we have $y_{m}<4(k+1)$. 
Suppose, on the contrary, that for some large $m \in \mathscr{H}, m$ and $m^{\prime}$ satisfy (3.9), (3.10) and (3.11), and suppose that $y_{m^{\prime}} \geqq 4(k+1)$. Then either

$$
\mu_{m^{\prime}} \geqq y_{m^{\prime}} / 2 \geqq 2(k+1)
$$

or

$$
\sigma_{m^{\prime}}-\tau_{m^{\prime}} \geqq y_{m^{\prime}} / 2 \geqq 2(k+1),
$$

and we shall show that both cases are impossible. We define $P(z), Q(z), h(z)$ and $H(z)$ as in (3.14)-(3.17), but with $m$ replaced by $m^{\prime}$, and note that if

then from Lemma 1 we have

$$
S=\mu_{m^{\prime}} \geqq y_{m^{\prime}} / 2
$$

on the circle

$$
\frac{S !}{(S-i) ! 2^{i}\left(3 \varrho_{m^{\prime}}\right)^{i}} \leqq\left|\frac{P^{(i)}(z)}{P(z)}\right| \leqq \frac{S ! 2^{i}}{(S-i) !\left(3 \varrho_{m^{\prime}}\right)^{i}}
$$

$$
C_{m^{\prime}}=\left\{z:\left|z-a_{m^{\prime}}\right|=3 \varrho_{m^{\prime}}\right\}
$$

for $i=1, \ldots, k+1$. Also, combining (3.13) and Claim 1, and noting that $r_{m} \leqq 3\left|a_{m^{\prime}}\right|$, we have, using Lemma 2 ,

$$
\left|\frac{h^{(i)}(z)}{h(z)}\right| \leqq c_{10} S^{i}\left(\log \left|a_{m^{\prime}}\right|\right)^{2 i}
$$

on $C_{m^{\prime}}$, for $i=1, \ldots, k+1$. But

$$
\psi(z)=P(z) h(z)\left[\alpha_{k}(z)\left[\frac{P^{(k)}(z)}{P(z)}+\ldots+\frac{h^{(k)}(z)}{h(z)}\right]+\ldots+\alpha_{0}(z)\right]
$$

and thus on $C_{m^{\prime}}$, using (3.22) and (3.23) we obtain from (3.24) (with $d$ denoting the maximum of the degrees of $\left.\alpha_{0}(z), \ldots, \alpha_{k}(z)\right)$

But

$$
\psi(z)=P(z) h(z)\left[\alpha_{k}(z) \frac{P^{(k)}(z)}{P(z)}+O\left(|z|^{d} S^{k} \frac{\left(\log \left|a_{m^{\prime}}\right|\right)^{2 k}}{\left(\varrho_{m^{\prime}}\right)^{k-1}}\right)\right] .
$$

$$
\frac{S !}{(S-k) ! S^{k}} \geqq 2^{-k}
$$

and thus, using (3.22), if $K(\varepsilon)$ is large enough in (3.2) we have

$$
\psi(z)=h(z) \alpha_{k}(z)(1+o(1)) P^{(k)}(z)
$$

on $C_{m^{\prime}}$. It follows from (3.25) and Rouché's theorem that $\psi(z)$ has the same number of zeros inside $C_{m^{\prime}}$ as $P^{(k)}(z)$; since the zeros of $P^{(k)}(z)$ lie in the convex hull of the set of zeros of $P(z)$, and hence in $D_{m^{\prime}}$, we conclude that $\psi(z)$ has $S-k$ zeros in the disc $B\left(a_{m^{\prime}}, 3 \varrho_{m^{\prime}}\right)$.

Similarly

$$
\begin{aligned}
\psi^{\prime}(z) & =P(z) h(z)\left[\alpha_{k}(z) \frac{P^{(k+1)}(z)}{P(z)}+\ldots+\alpha_{0}^{\prime}(z)\right] \\
& =(1+o(1)) P^{(k+1)}(z) \alpha_{k}(z) h(z)
\end{aligned}
$$


on $C_{m^{\prime}}$, and thus

But then

$$
\tau_{m^{\prime}}=\mu_{m^{\prime}}-(k+1)
$$

$$
\sigma_{m^{\prime}} \geqq y_{m^{\prime}} / 2>0
$$

and so $Q(z) \not \equiv 1$ in (3.17). Moreover, inside the circle $C_{m^{\prime}}$ we have, using (3.3), (3.13), Claim 1 and (3.26),

$$
\begin{aligned}
\log |\psi(z)-1| & =\log |H(z)|+\log |Q(z)| \\
& \leqq c_{5} T\left(r_{m}, \psi-1\right) \\
& +c_{6} \sigma_{m^{\prime}} \log r_{m} \\
& +\sigma_{m^{\prime}} \log 6 \varrho_{m^{\prime}} \\
& \left.\leqq c_{11} \sigma_{m^{\prime}} \log \left|a_{m^{\prime}}\right|\right)^{2}+\sigma_{m^{\prime}} \log 6 \varrho_{m^{\prime}}<0
\end{aligned}
$$

if $K(\varepsilon)$ is large enough in (3.2). But this implies that $\psi(z)$ has no zeros inside $C_{m^{\prime}}$, contradicting the conclusion that followed (3.25).

On the other hand, suppose that $y_{m^{\prime}} \geqq 4(k+1)$, and that

But then, since

$$
\sigma_{m^{\prime}}-\tau_{m^{\prime}} \geqq y_{m^{\prime}} / 2 \text {. }
$$

$$
\psi^{\prime}(z)=Q^{\prime}(z) H(z)+Q(z) H^{\prime}(z)
$$

it follows from Lemmas 1 and 2, and (3.3), (3.13), and Claim 1 that

$$
\psi^{\prime}(z)=Q^{\prime}(z) H(z)(1+o(1))
$$

on $C_{m^{\prime}}$, and hence that $\sigma_{m^{\prime}}-\tau_{m^{\prime}}=1$. This establishes Claim 2 .

Claim 3. We have

$$
T(r, f)=O(\log r)^{2}
$$

and in particular, for large $m$,

$$
T\left(r_{m}, f\right)+T\left(r_{m}, \psi\right) \leqq c_{12}\left(\log \left|a_{m}\right|\right)^{2} .
$$

This follows immediately from (3.8) and Claim 2.

Claim 4. For all large $m$, we have $\mu_{m}<2(k+1)$.

For, suppose that $\mu_{m} \geqq 2(k+1)$ for some large $m$. Then on the circle

$$
C_{m}=\left\{z:\left|z-a_{m}\right|=3 \varrho_{m}\right\}
$$

we have, defining $P, Q, h$ and $H$ as in (3.14) to (3.17) and setting $S=\mu_{m}$,

$$
\frac{S !}{(S-i) ! 2^{i}\left(3 \varrho_{m}\right)^{i}} \leqq\left|\frac{P^{(i)}(z)}{P(z)}\right| \leqq \frac{S ! 2^{i}}{(S-i) !\left(3 \varrho_{m}\right)^{i}}
$$


for $i=1, \ldots, S$, which of course is just (3.22) with $m^{\prime}$ replaced by $m$. Also, from Claim 1, (3.28) and Lemma 2 we have, for $i=1, \ldots, k$,

$$
\left|\frac{h^{(i)}(z)}{h(z)}\right| \leqq c_{13} S^{i}\left(\log \left|a_{m}\right|\right)^{2 i}
$$

on $C_{m}$. But then, on $C_{m}$, with $d$ denoting the maximum of the degrees of $\alpha_{0}(z), \ldots, \alpha_{k}(z)$,

$$
\begin{aligned}
\psi(z) & =P(z) h(z)\left[\alpha_{k}(z) \frac{P^{(k)}(z)}{P(z)}+O\left(|z|^{d} S^{k} \frac{\left(\log \left|a_{m}\right|\right)^{2 k}}{\varrho_{m}^{k-1}}\right)\right] \\
& =\alpha_{k}(z) h(z) P^{(k)}(z)(1+o(1))
\end{aligned}
$$

using (3.29) and (3.30) and noting that $(S-k)>S / 2$. Hence $\psi(z)$ has $S-k$ zeros in $B\left(a_{m}, 3 \varrho_{m}\right)$ and so, by Rouché's theorem, $\psi(z)-1$ has at least $S-k$ zeros in $D_{m}$ since by Theorem $\mathrm{C} \psi(z)$ is large outside an $\varepsilon$-set. But then, using Claim 1 and (3.28), we have, in $B\left(a_{m}, 3 \varrho_{m}\right)$,

$$
\begin{aligned}
\log |\psi(z)-1| & =\log |H(z)|+\log |Q(z)| \\
& \leqq c_{14}\left(\log \left|a_{m}\right|\right)^{2}+c_{6} \sigma_{m} \log r_{m} \\
& +\sigma_{m} \log 6 \varrho_{m}<0
\end{aligned}
$$

if $K(\varepsilon)$ is large enough in (3.2), and we have a contradiction.

Claim 5. For large $m$, if $\sigma_{m}>0$, then $\mu_{m} \geqq \sigma_{m}+k$.

We note first that if $\mu_{m}=0$ then by Theorem C and Lemma 3, $\psi(z)$ is large in the disc $B\left(a_{m}, \varepsilon\left|a_{m}\right| / 2\right)$ and hence $\sigma_{m}=0$. Thus, if $\sigma_{m}>0$, we set

$$
c=\min \left\{\mu_{m}, k\right\} \geqq 1
$$

and we write

$$
\psi(z)=P(z) h(z)\left[\frac{P^{(c)}(z)}{P(z)} L(z)+B(z)\right]
$$

where $P, h, Q$ and $H$ are defined as in (3.14) to (3.17) and $B(z), L(z)$ are as follows. We have

if $c<k$, and

$$
L(z)=\left(\alpha_{k}(z) \frac{k !}{c !(k-c) !} \frac{h^{(k-c)}(z)}{h(z)}+\ldots+\alpha_{c}(z)\right)
$$

$$
L(z)=\alpha_{k}(z)
$$

if $c=k$. Thus, if we regard $\psi(z) / f(z)$ as a linear combination of terms $P^{(i)}(z) / P(z)$ with $i \leqq c$, then $L(z)$ is the coefficient of $P^{(c)}(z) / P(z)$. Accordingly, $B(z)$ is a linear combination of terms $P^{(i)}(z) / P(z)$ with $i<c$, and with coefficients which are sums of terms $\gamma_{i j}(z) h^{(j)}(z) / h(z)$ where each $\gamma_{i j}(z)$ is a constant multiple of one of $\alpha_{0}(z), \ldots, \alpha_{k}(z)$. 
On the circle $C_{m}$, by Lemmas 1 and 3, and Claim 4,

$$
|B(z)|=O\left(|z|^{d} \varrho_{m}^{1-c}\right)
$$

where $d$ as before is the maximum of the degrees of $\alpha_{0}(z), \ldots, \alpha_{k}(z)$. We may also apply the second part of Lemma 3 to $h(z)$ (since for all $r, n(r, 1 / h)$ and $n(r, 1 / f)$ differ by at most $2 k+1$ ) to conclude that, on the circle $C_{m}$,

$$
|L(z)| \geqq c_{15}|z|^{-k} \text {. }
$$

Combining (3.2), (3.29), (3.30), (3.31), (3.32) and (3.33) we see that

$$
\psi(z)=h(z) P^{(c)}(z) L(z)(1+o(1))
$$

on the circle $C_{m}$ and hence that $\psi(z)$ has $v_{m}$ zeros in $B\left(a_{m}, 3 \varrho_{m}\right)$, where

$$
v_{m}=\max \left\{0, \mu_{m}-k\right\},
$$

since $h(z)$ and $L(z)$ do not vanish on or inside $C_{m}$.

Thus, if $\sigma_{m}>0$, we must have $\mu_{m}>k$. For otherwise $P^{(c)}(z)$ is a non-zero constant and $v_{m}=0$; also on the circle $\left\{z:\left|z-a_{m}\right|=\varepsilon\left|a_{m}\right| / 4\right\}$ we have

$$
\log |h(z)| \geqq \log |f(z)|-k \log \left|a_{m}\right|-O(1)
$$

and so, by Theorem $\mathrm{C}$ and the minimum principle $h(z)$ is large in $\left|z-a_{m}\right| \leqq 3 \varrho_{m}$. From (3.34), $\psi(z)$ must be large on $C_{m}$, and again by the minimum principle, we conclude that $\sigma_{m}=0$, which is a contradiction.

Thus $\sigma_{m}>0$ implies that $\mu_{m} \geqq k+1$. Now, we may apply the above reasoning ((3.31) to (3.35)) to $\psi^{\prime}(z)$ to conclude that

$$
\tau_{m}=\max \left\{\mu_{m}-(k+1), 0\right\} .
$$

Also,

$$
\tau_{m}=\sigma_{m}-1
$$

since on $C_{m}$,

$$
\begin{aligned}
\psi^{\prime}(z) & =H(z) Q(z)\left(\frac{Q^{\prime}(z)}{Q(z)}+\frac{H^{\prime}(z)}{H(z)}\right) \\
& =H(z) Q^{\prime}(z)(1+o(1))
\end{aligned}
$$

using (3.2), Claim 1, (3.28) and Lemmas 1 and 2. Combining (3.36) and (3.37) we see that if $\sigma_{m}>1$ then

$$
\tau_{m}=\mu_{m}-(k+1) \text { and } \sigma_{m}=\mu_{m}-k .
$$

Claim 5 is now proved.

We may now conclude the proof of Theorem 1 by obtaining a contradiction. From Claims 4 and 5 we have, for large $m$, if $\sigma_{m}>0$,

$$
\sigma_{m} \leqq \mu_{m}-k \leqq \frac{3}{4} \mu_{m}
$$


and thus

$$
N\left(r, \frac{1}{\psi-1}\right)<\frac{7}{8} N\left(r, \frac{1}{f}\right)
$$

for large $r$. But for large $m$, using Lemma 3 and Theorem $\mathrm{C}$, and recalling that the annulus $A_{m}$ as defined by (3.5) meets none of the discs $D_{n}$ we have

$$
\begin{aligned}
N\left(r_{m}, \frac{1}{f}\right) & <m\left(r_{m}, f\right)+O(1) \\
& <m\left(r_{m}, \psi\right)+m\left(r_{m}, \frac{f}{\psi}\right)+O(1) \\
& <m\left(r_{m}, \psi-1\right)+O\left(\log r_{m}\right) \\
& <N\left(r_{m}, \frac{1}{\psi-1}\right)+O\left(\log r_{m}\right) \\
& <\frac{15}{16} N\left(r_{m}, \frac{1}{f}\right)
\end{aligned}
$$

using (3.38), which is impossible.

\section{Proof of Theorem 2}

We set

$$
f(z)=\prod_{n=1}^{\infty}\left(1-\frac{z}{a_{n}}\right)
$$

where $a_{n+1}>a_{n}$ and the $a_{n}$ lie in the set $\left\{q, q^{2}, q^{3}, \ldots\right\}$ for some $q>81$. We will make the actual choice of the $a_{n}$ later.

Consider a large zero, $z_{0}$ say, of $f^{\prime}(z)$, which must be real and positive ([9], p. 266). Suppose that $a_{N}$ is the nearest zero of $f(z)$ to $z_{0}$, choosing the larger if $z_{0}$ is equidistant from two zeros of $f(z)$. Now

$$
\frac{f^{\prime}\left(z_{0}\right)}{f\left(z_{0}\right)}=\sum_{n=1}^{\infty} \frac{1}{z_{0}-a_{n}}=0
$$

But

$$
\sum_{n>N} \frac{1}{a_{n}-z_{0}} \leqq \frac{1}{a_{N+1}-z_{0}}+\sum_{k=1}^{\infty} \frac{1}{\left(q^{k}-1\right) z_{0}} \leqq \frac{c_{1}}{z_{0}}
$$

say. Also, for $n<N, a_{n}<z_{0}$ and so

$$
\sum_{n=1}^{N-1} \frac{1}{z_{0}-a_{n}}>\frac{N-1}{z_{0}}
$$


Combining (4.2), (4.3) and (4.4) we obtain

and so

$$
\frac{1}{\left|z_{0}-a_{N}\right|}>\frac{N-1}{z_{0}}-\frac{c_{1}}{z_{0}}
$$

$$
\left|z_{0}-a_{N}\right|<c_{2} z_{0}\left(n\left(z_{0}, \frac{1}{f}\right)\right)^{-1},
$$

say. Thus, given a small, positive $\varepsilon$, all large enough zeros of $f^{\prime}(z)$ lie in the union of the discs $B\left(a_{n}, \varepsilon a_{n} / 2\right)$, and, observing that $T(r, f)=O(\log r)^{2}$, we see from Theorem $\mathrm{C}$ that all large 1-points of $f^{\prime}(z)$ lie in the union of the discs $B\left(a_{n}, \varepsilon a_{n}\right)$. Thus, for large $n$, the annuli $A_{n}=\left\{z: q^{1 / 4} a_{n} \leqq|z| \leqq q^{1 / 2} a_{n}\right\}$ are free of zeros and 1-points of $f^{\prime}(z)$; also, by Lemma 4 there must exist level curves $J_{n}$ each closing in $A_{n}$ on which $|f(z)|=M\left(q^{1 / 4} a_{n}, f\right)$. But then (see [9], p. 122) $f(z)$ and $f^{\prime}(z)$ have the same number of zeros in the region between $J_{n}$ and $J_{n+1}$, and so, for large $n$, $f^{\prime}(z)$ has exactly one zero, $x_{n}$ say, and one 1-point, $z_{n}$ say, in the disc $B\left(a_{n}, \varepsilon a_{n}\right)$. Now, $f\left(a_{n}\right)=f\left(a_{n+1}\right)=0$, and since

$$
\frac{d}{d x}\left(\frac{f^{\prime}(x)}{f(x)}\right)=-\sum_{k=1}^{\infty} \frac{1}{\left(x-a_{k}\right)^{2}}<0
$$

on $\left(a_{n}, a_{n+1}\right)$ we see that $f(x)$ has exactly one maximum or minimum on each interval $\left(a_{n}, a_{n+1}\right)$. Suppose now that $n$ is large and that $x_{n}$ is a maximum point for $f(x)$ on $\left(a_{n-1}, a_{n+1}\right)$. If we have $x_{n}>a_{n}$ then $f^{\prime}\left(a_{n}\right)>0$ and $f^{\prime}\left((1-\varepsilon) a_{n}\right)>10$ (using Theorem C) and so $(1-\varepsilon) a_{n}<z_{n}<x_{n}$. Similarly, if $x_{n}<a_{n}$ then $f^{\prime}\left((1-\varepsilon) a_{n}\right)>10$ and $(1-\varepsilon) a_{n}<z_{n}<x_{n}$. On the other hand, if $x_{n}$ is a minimum point for $f(x)$ on $\left(a_{n-1}, a_{n+1}\right)$ then $x_{n}<z_{n}<(1+\varepsilon) a_{n}$. Since $\left|f\left(x_{n}\right)\right|>a_{n}^{10}$, say, we obtain, by integration,

$$
\left|f\left(z_{n}\right)\right|>z_{n}^{2}
$$

in either case.

Thus we have shown that for all large 1-points $z_{n}$ of $f^{\prime}(z), z_{n}>0$ and $\left|f\left(z_{n}\right)\right|$ is large. But then $f^{\prime}\left(z_{n}\right) / f\left(z_{n}\right)$ is small and

For $k<n$,

while (compare 4.3)

$$
\frac{1}{\left|z_{n}-a_{n}\right|} \leqq-\frac{o(1)}{z_{n}}+\left|\sum_{k \neq n} \frac{1}{z_{n}-a_{k}}\right| .
$$

say. Thus (4.5) yields

$$
\left|z_{n}-a_{k}\right|>\left(1-q^{-1 / 2}\right) z_{n}
$$

$$
\left|\sum_{k>n} \frac{1}{z_{n}-a_{k}}\right| \leqq \frac{c_{3}}{z_{n}},
$$

$$
\frac{1}{\left|z_{n}-a_{n}\right|} \leqq \frac{c_{4}}{z_{n}} n\left(z_{n}, \frac{1}{f}\right) \leqq \frac{c_{5}}{a_{n}} n\left(a_{n}, \frac{1}{f}\right)
$$


for some $c_{4}, c_{5}$, and setting $c_{6}=\max \left\{c_{4}, c_{5}\right\}$ we need only choose the points $a_{n}$ from the set $\left\{q, q^{2}, q^{3}, \ldots\right\}$ in such a way as to ensure that $c_{6} n(r, 1 / f) \leqq h(r)$ for all $r$. The set $\left\{b_{m}\right\}$ is then the set of large $a_{n}$ and $z_{n}$ points, arranged in order of magnitude, and we have

This proves Theorem 2 .

$$
b_{m+1}-b_{m} \geqq b_{m}\left(h\left(b_{m}\right)\right)^{-1} \text {. }
$$

Remark. The author wishes to thank the referee for some helpful comments, and for pointing out a number of minor errors.

\section{References}

[1] AhLfors, L. V.: Complex analysis. An introduction to the theory of analytic functions of one complex variable. Second edition. - McGraw-Hill Book Co., New York-Toronto, Ont.-London, 1966.

[2] Anderson, J. M., I. N. BAKer, and J. Clunie: The distribution of values of certain entire and meromorphic functions. - Math. Z. 178, 1981, 509-525.

[3] Baker, I. N., and L. S. O. Liverpool: Picard sets for entire functions. - Math. Z. 126, 1972, $230-238$.

[4] BAKer, I. N., and L. S. O. LiverPool: Further results on Picard sets of entire functions. Proc. London Math. Soc. (3) 26, 1973, 82-98.

[5] Hayman, W. K.: Picard values of meromorphic functions and their derivatives. - Ann. of Math. (2) 70, 1959, 9-42.

[6] Hayman, W. K.: Slowly growing integral and subharmonic functions. - Comment. Math. Helv. $34,1960,75-84$.

[7] Hayman, W. K.: Meromorphic functions. - Oxford Mathematical Monographs. Clarendon Press, Oxford, 1964.

[8] Langley, J. K.: Some exceptional sets for meromorphic functions and their derivatives. - Ph. D. Thesis, University of London, 1983.

[9] Titchmarsh, E. C.: The theory of functions. - Clarendon Press, Oxford, 1932.

[10] ToppILA, S.: Some remarks on the value distribution of entire functions. - Ark. Mat. 9, 1971, $1-9$.

Research carried out while a visiting lecturer at:

University of Illinois

Department of Mathematics

Urbana, IL 61801

USA

Current address:

University of St. Andrews

The Mathematical Institute

North Haugh, St. Andrews

KY 16 955, Scotland

Received 28 August 1984 